\title{
Psychological Distress Among Lebanese Women During the COVID-19 Confinement: Any Mediating Effect of Violence?
}

\section{Chadia Haddad ( $\square$ chadia_9@hotmail.com )}

Psychiatric Hospital of the Cross: Hopital Psychiatrique de la Croix https://orcid.org/0000-0003-2413-2684

\section{Diana Malaeb}

Lebanese International University

Hala Sacre

INSPECT

\section{Sandrella Bou Malhab}

Lebanese University: Universite Libanaise

\section{Dalia Khachman}

Lebanese university

Nathalie Lahoud

Lebanese University: Universite Libanaise

Joelle Azzi

Lebanese University: Universite Libanaise

Pascale Salameh

Lebanese University: Universite Libanaise

\section{Research article}

Keywords: violence, women, anxiety, stress, depression, psychological distress.

Posted Date: March 3rd, 2021

DOI: https://doi.org/10.21203/rs.3.rs-285748/v1

License: (c) (i) This work is licensed under a Creative Commons Attribution 4.0 International License. Read Full License 


\section{Abstract}

Background: Violence among women is associated with poor psychological health and increased internalizing symptoms, such as depression, stress, and anxiety. The primary objective was to evaluate the association of marital conflict, pregnancy status, socioeconomic status, and stressful life events with violence, depression, anxiety, and stress. The secondary objective was to evaluate the mediating effect of violence on psychological distress.

Methods: A cross-sectional study was conducted among 369 women between June 8 and August 1, 2020. The questionnaire was developed on Google Forms and distributed online, using the snowball technique.

Results: Higher marital conflict (Beta=0.159), financial difficulties (Beta=2.572), a history of child abuse (Beta=2.546), and higher violence scores (Beta=0.121) were significantly associated with higher depression scores. Higher marital conflict (Beta=0.358) and having financial difficulties (Beta=3.859) were significantly associated with higher anxiety scores. Higher marital conflict $(B e t a=0.070)$ and financial difficulties (Beta $=1.483$ ) were significantly associated with higher perceived stress scores. Composite abuse scale partially mediated the association between pregnancy status $(25.28 \%)$, financial difficulties $(7.71 \%)$, history of child abuse $(21.92 \%)$, abuse by a family member $(50.50 \%)$, and anxiety scale. Also, the composite abuse scale partially mediated the association between marital conflict $(21.42 \%)$, financial difficulties $(14.17 \%)$, history of child abuse $(20.05 \%)$, abuse by a family member (33.57\%), and depression scale.

Conclusion: Our main findings suggest that violence mediated the association between pregnancy status, marital conflicts, financial difficulties, and depression and anxiety but did not mediate between these factors and stress. Further studies are needed to understand better the factors that mediate the relations between domestic violence and mental well-being among Lebanese women.

\section{Introduction}

Depression, anxiety, and stress are now the leading mental health disorders causing disability, and having a high burden on the health care budget $(1,2)$. Severe mental health problems are associated with emotional, cognitive, and social abilities that can lead to the vast majority of detrimental consequences $(3,4)$. Depression is much more common in women than in men due to hormonal changes at different stages during puberty, prior to menstruation, following pregnancy, and at perimenopause (5). A higher prevalence of depression among women is attributed not only to the biological factor but also to environmental and social factors (6-8). Previous literature supported that other risk factors increased the propensity of depression among married women, such as marital conflicts, lack of social support, and marital dissatisfaction (9-11). In addition to the influence of the social environment, other potential risk factors also increased the risk of depression, including a low level of education and financial difficulties $(12,13)$.

Married women with low income are more likely to encounter financial difficulties, relationship problems, unstable health, and unemployment, which can contribute to the onset of depression (14). A poor relationship with the partner causes intimate partner violence and stress in the marital life; also, and husbands busy with their professions or having extramarital affairs increase the risk for depression in women $(7,15-17)$. On the other hand, enhanced marital satisfaction has been associated with a lower propensity to develop depressive symptoms (18). Married women undergoing domestic violence, work stress, traumatic experiences, and abused conditions had a higher risk of depression (19).

In addition to depression, women are more likely than men to experience anxiety due to biological, economic, and social factors (20). The hormonal fluctuations associated with menstruation and pregnancy increase the vulnerability of women to mental distress, such as anxiety (21), also affected by social factors, including poverty, conflict at work or with the partner, prolonged working hours, and job dissatisfaction $(22,23)$. Women of low socioeconomic status continually fear that they will not be able to meet their daily needs and worry about the future, which limits their recreational activities (21). Hence, poverty, combined with a lack of social support, induce a higher risk of anxiety and depressive disorders in women. 
Stress, another mental health disorder, is a highly prevalent condition in women, particularly during pregnancy (24). Pregnant women are highly prone to stress due to the feeling of imbalance when they cannot cope with demands and worries (25). Other influential factors of increased risk of stress among women include low socioeconomic status, exposure to violence, stressful life events, lack of social support, and conflicts (26-28).

Previous literature supports the fact that violence among women is related to several factors, including low socioeconomic level, previous violence in the family, pregnancy status, and social isolation (29). Furthermore, women exposed to violence had poor psychological health and increased internalizing symptoms, such as depression, stress, and anxiety $(30,31)$. Previous studies reported various mediating factors for violence. However, only a few examined the mediating effect of violence on psychological distress in women $(32,33)$. Our research uses a conceptual model based on a study done by Ceballo et al. (Fig. 1) (29).

While these problems are reported in the literature and have different health effects, no studies have yet evaluated the magnitude of depression, anxiety, and stress among Lebanese women. Therefore, our primary objective was to assess the association of marital conflict, pregnancy status, socioeconomic status, and stressful life events with violence, depression, anxiety, and stress. Our secondary objective was to evaluate the mediating effect of violence on psychological distress.

\section{Methods}

\section{Study design and sampling}

A cross-sectional online study conducted between June 8 and August 1, 2020, enrolled a total of 369 women. The questionnaire used was developed on Google Forms and distributed on social media and WhatsApp groups, using the snowball technique; it required 40 minutes to complete. Eligibility criteria were the following: married women aged between 18 and 51, currently living with their partner, and with internet access. Single, widowed, or divorced women, and those with a fertility problem, were excluded. The inclusion criteria were stated in the consent form at the beginning of the survey. Participation in this study was anonymous and voluntary, and participants received no compensation in return.

\section{Sample size calculation}

The Epi info software (Centers for Disease Control and Prevention, Epi Info ${ }^{\text {TM}}$ ) calculated a minimum sample of 233 participants, taking into account a Lebanese female population of 2,294,260 (34), a prevalence of 10.9\% women with depression, according to a previous study (35), a confidence level of $95 \%$, and adding a $4 \%$ margin of error. A sample of 500 women was targeted to allow for missing values. The final sample size consisted of 369 participants.

\section{Translation procedure}

A forward and backward translation was conducted for all the items of the questionnaire. One translator did the translation from English into Arabic, and a second one performed the back translation. Discrepancies between the two English versions were resolved by consensus.

\section{Questionnaire}

The online questionnaire consisted of two sections, including closed-ended questions available in English and Arabic. The questionnaire was developed for this study and it is provided as Additional File 1. The first one assessed the sociodemographic details of participants (age, educational level, the region of residence, religion, working status, monthly income, smoking and alcohol status, and physical activity). The monthly income was divided into four levels: no income, low $<1,000$ USD, intermediate $1,000-2,000$ USD, and high income $>2,000$ USD.

The second section consisted of the following measures:

\section{Household crowding index}


The household crowding index was calculated by dividing the number of persons living in the household by the number of rooms, excluding bathrooms and kitchen, to assess socioeconomic status (36).

\section{Stressful life events}

Negative life events were assessed using three dichotomous questions (Yes/No) about financial difficulties, history of child abuse, and history of family member abuse.

\section{Marital conflict}

The Relationship Dynamics Scale was utilized to measure the frequency of conflicts in relationships (37). The scale consists of eight items scored on a 5 -point Likert scale, $(1=$ Never or almost never to $5=$ Always $)(37)$. A higher score indicates a higher conflict between partners.

\section{Pregnancy status}

The current pregnancy status was assessed using binary questions (Yes/No). The current pregnancy status reflects the ability of women to choose to reproduce or be coerced into an unwanted pregnancy.

\section{Partner violence}

Violence was assessed using the Composite Abuse Scale (Revised) - Short Form (CASR-SF). This 15-item scale evaluates the existence, extent, and severity of physical, sexual, or psychological abuse (38). The total score is calculated by summing the 15 responses. Items are graded on a Likert scale from 1 to 6 , where a higher score indicates a higher intensity/occurrence of abuse. The author of the questionnaire, Professor Marilyn Ford-Gilboe, granted permission to use the scale.

\section{Psychological distress}

Stress, anxiety, and depression were measured using three scales, reflecting psychological distress among women. The Perceived Stress Scale-4 (PSS-4) evaluated the occurrence and intensity of stress. It consists of four questions graded on a Likert scale from 1 to 5 . The total score was calculated by summing the four items, ranging from 0 to 16 , with higher scores indicating a higher intensity/occurrence of perceived stress (39). The Lebanese anxiety scale (LAS; developed and validated in Lebanon) is a 10-item tool graded on a Likert scale from 1 to 5 . It assessed the occurrence and intensity of anxiety. The total score was obtained by summing all the responses, with higher scores indicating higher anxiety (40). The PHQ-9, validated in Lebanon (41), assesses the level of depression. It consists of 9 items graded on a Likert scale from 1 to 4 . The PHQ-9 score can range from 0 to 27 , with higher scores indicating a more severe depression (42).

All the scales used in this study do not require any license for their use except the CASR-SF where a permission from the author was granted.

\section{Statistical analysis}

Data were analyzed on SPSS software version 25 (SPSS Inc., Chicago, IL, USA). A descriptive analysis was performed, using the absolute frequency and percentages for categorical variables and mean and standard deviation for quantitative measures. Student t-test and ANOVA F tests were used to assess the association of continuous variables with two or more levels, and Pearson correlation was used for linear correlation between continuous variables. In the multivariable analysis, four linear regressions were performed, taking stress, anxiety, depression, and violence as the dependent variables, meeting all the assumptions for normality and homoscedasticity. Regressions took into account the variables with a value of $P<0.2$ in the bivariate analysis to minimize residual confounding.

The PROCESS SPSS Macro version 3.4 model four was used to evaluate the mediation of violence using the CASR-SF through three steps. Step 1 determined the regression coefficient for the effect of marital conflict, household crowding index, pregnancy status, financial difficulties, history of child abuse, and abuse by a family member. Step 2 examined the association between violence and psychological distress (stress, anxiety, and depression), and Step 3 estimated the direct effect of each of the marital 
conflict, household crowding index, pregnancy status, financial difficulties, history of child abuse, and abuse by a family member on psychological distress. Each independent variable was entered in a separate model since the variables were not highly correlated. The macro generated bias-corrected bootstrapped $95 \%$ confidence intervals $(\mathrm{Cl})$ to test the significance of the indirect effect $(43,44)$. Mediation was considered significant when the $\mathrm{Cl}$ around the indirect effect did not include zero $(44)$. The mediation effect was calculated by dividing the indirect effect of $X$ by the direct effect of $Y$. The covariates included in the mediation model were those that showed significant associations with psychological distress in the bivariate analysis.

Statistical significance was set at a value of $\mathrm{P}<0.05$.

\section{Results}

\section{Sample description}

The mean age of women was $32.5 \pm 6.4$ years. The majority $(83.9 \%)$ had a university level of education, $59.9 \%$ were employed, $23.3 \%$ had no income, and $42.5 \%$ practiced physical activities. Only $31.2 \%$ of them were smokers, and $10.8 \%$ consumed alcohol. The mean duration of confinement was $71.0 \pm 42.8$ days, and the mean fear of poverty was $5.8 \pm 3.2$.

\section{Bivariate analysis: correlates of psychological scales}

A significantly higher mean violence score was found in non-pregnant as compared to pregnant women $\left(M_{\text {non-pregnant women }}=2.18\right.$ vs. $\left.M_{\text {pregnant women }}=0.39, \mathrm{P}=0.025\right)$, those with a history of family member abuse $\left(M_{\text {Yes }}=8.15\right.$ vs. $\left.M_{\text {No }}=1.51, P<0.001\right)$ or a history of child abuse $\left(M_{\mathrm{Yes}}=4.89\right.$ vs. $\left.M_{\mathrm{No}}=1.45, \mathrm{P}<0.001\right)$. Additionally, older age $(r=0.149)$, longer duration of marriage $(r=$ $0.127)$, higher marital conflict $(r=0.510)$, higher stress $(r=0.123)$, depression $(r=0.259)$, and anxiety $(r=0.141)$ were significantly associated with higher violence.

A significantly higher mean depression score was found in non-employed as compared to employed women $\left(M_{\text {non-employed }}=7.95\right.$ vs. $\left.M_{\text {employed }}=6.41, \mathrm{P}=0.031\right)$, those with financial difficulties $\left(M_{\text {Yes }}=8.74\right.$ vs. $\left.M_{\text {No }}=5.66, P<0.001\right)$, history of child abuse $\left(M_{\text {Yes }}=10.74\right.$ vs. $\left.M_{\text {No }}=6.35, \mathrm{P}<0.001\right)$, or history of family member abuse $\left(M_{\text {Yes }}=12.54\right.$ vs. $\left.M_{\text {No }}=6.61, P<0.001\right)$. Additionally, higher marital conflict $(r=0.353)$, higher stress $(r=0.447)$, anxiety $(r=0.785)$, and violence $(r=0.259)$ were significantly associated with higher depression.

A significantly higher mean anxiety score was found in non-pregnant as compared to pregnant women $\left(M_{\text {non-pregnant women }}=14.73\right.$ vs. $\left.M_{\text {pregnant women }}=12.12, \mathrm{P}=0.034\right)$, those unemployed $\left(M_{\text {non-employed }}=16.01\right.$ vs. $\left.M_{\text {employed }}=13.39, \mathrm{P}=0.004\right)$, with financial difficulties $\left(M_{\text {Yes }}=17.22\right.$ vs. $\left.M_{\text {No }}=12.22, \mathrm{P}<0.001\right)$, history of child abuse $\left(M_{\text {Yes }}=17.95\right.$ vs. $\left.M_{\text {No }}=13.80, P<0.001\right)$, or abuse by a family member $\left(M_{\text {Yes }}=19.19\right.$ vs. $\left.M_{\text {No }}=14.08, P=0.003\right)$. Moreover, higher marital conflict $(r=0.338)$, higher stress $(r=0.503)$, depression $(r=0.785)$, and violence $(r=0.141)$ were significantly associated with higher anxiety.

A significantly higher mean stress score was found in those with financial difficulties $\left(M_{\text {Yes }}=8.20\right.$ vs. $\left.M_{\text {No }}=6.68, P<0.001\right)$. Also, higher marital conflict $(r=0.211)$, higher depression $(r=0.447)$, anxiety $(r=0.503)$ and violence $(r=0.123)$ were significantly associated with higher stress (Table 1 ). 
Table 1

Bivariate analysis taking the psychological scales as the dependent variables

\begin{tabular}{|c|c|c|c|c|}
\hline & Total violence score & Depression score & Anxiety score & Stress score \\
\hline & Mean \pm SD & Mean \pm SD & Mean \pm SD & Mean \pm SD \\
\hline \multicolumn{5}{|l|}{ Pregnancy status } \\
\hline Yes & $0.39 \pm 1.20$ & $6.07 \pm 6.03$ & $12.12 \pm 6.47$ & $6.83 \pm 2.41$ \\
\hline No & $2.18 \pm 6.14$ & $7.15 \pm 5.36$ & $14.73 \pm 7.45$ & $7.42 \pm 2.65$ \\
\hline P-value & 0.025 & 0.050 & 0.034 & 0.233 \\
\hline \multicolumn{5}{|l|}{ Financial difficulties } \\
\hline Yes & $2.91 \pm 7.53$ & $8.74 \pm 5.75$ & $17.22 \pm 7.54$ & $8.20 \pm 2.42$ \\
\hline No & $1.23 \pm 3.84$ & $5.66 \pm 4.76$ & $12.22 \pm 6.46$ & $6.68 \pm 2.58$ \\
\hline P-value & 0.311 & $<0.001$ & $<0.001$ & $<0.001$ \\
\hline \multicolumn{5}{|l|}{ Working status } \\
\hline Yes & $1.45 \pm 4.44$ & $6.41 \pm 4.91$ & $13.39 \pm 6.59$ & $7.20 \pm 2.53$ \\
\hline No & $2.76 \pm 7.39$ & $7.95 \pm 6.05$ & $16.01 \pm 8.21$ & $7.58 \pm 2.75$ \\
\hline P-value & 0.320 & 0.031 & 0.004 & 0.292 \\
\hline \multicolumn{5}{|l|}{ Monthly income } \\
\hline No income & $2.04 \pm 5.19$ & $7.53 \pm 5.69$ & $16.58 \pm 8.06$ & $7.73 \pm 2.45$ \\
\hline Low & $2.28 \pm 6.13$ & $7.49 \pm 5.30$ & $14.17 \pm 6.58$ & $7.29 \pm 2.52$ \\
\hline Intermediate & $1.33 \pm 4.13$ & $6.28 \pm 4.92$ & $13.06 \pm 6.78$ & $6.94 \pm 2.76$ \\
\hline High & $2.61 \pm 8.50$ & $6.87 \pm 6.07$ & $13.84 \pm 7.82$ & $7.61 \pm 2.75$ \\
\hline P-value & 0.885 & 0.171 & 0.007 & 0.261 \\
\hline \multicolumn{5}{|l|}{ History of child abuse } \\
\hline Yes & $4.89 \pm 7.51$ & $10.74 \pm 6.09$ & $17.95 \pm 7.04$ & $7.54 \pm 2.51$ \\
\hline No & $1.45 \pm 5.32$ & $6.35 \pm 5.04$ & $13.80 \pm 7.27$ & $7.32 \pm 2.65$ \\
\hline P-value & $<0.001$ & $<0.001$ & $<0.001$ & 0.480 \\
\hline \multicolumn{5}{|c|}{ History of family member abuse } \\
\hline Yes & $8.15 \pm 11.74$ & $12.54 \pm 6.81$ & $19.19 \pm 8.61$ & $7.85 \pm 2.69$ \\
\hline No & $1.51 \pm 4.84$ & $6.61 \pm 5.10$ & $14.08 \pm 7.17$ & $7.32 \pm 2.62$ \\
\hline P-value & $<0.001$ & $<0.001$ & 0.003 & 0.331 \\
\hline Age & 0.149 & -0.066 & -0.038 & -0.037 \\
\hline$P$-value & 0.004 & 0.209 & 0.466 & 0.473 \\
\hline Household crowding index & -0.063 & 0.014 & 0.018 & 0.030 \\
\hline P-value & 0.231 & 0.791 & 0.733 & 0.560 \\
\hline Marital conflict & 0.510 & 0.353 & 0.338 & 0.211 \\
\hline P-value & $<0.001$ & $<0.001$ & $<0.001$ & $<0.001$ \\
\hline
\end{tabular}




\begin{tabular}{|lllll|}
\hline & Total violence score & Depression score & Anxiety score & Stress score \\
\hline Duration of marriage & 0.127 & -0.068 & -0.046 & -0.017 \\
\hline$P$-value & 0.014 & 0.192 & 0.380 & 0.741 \\
\hline Number of pregnancies & 0.074 & -0.051 & -0.023 & -0.011 \\
\hline$P$-value & 0.158 & 0.332 & 0.666 & 0.831 \\
\hline Number of children & 0.080 & 0.002 & 0.027 & 0.036 \\
\hline$P$-value & 0.163 & 0.978 & 0.637 & 0.529 \\
\hline Stress & 0.123 & 0.447 & 0.503 & - \\
\hline$P$-value & $\mathbf{0 . 0 1 8}$ & $<0.001$ & $<0.001$ & \\
\hline Depression & 0.259 & - & 0.785 & 0.447 \\
\hline$P$-value & $<0.001$ & & $<0.001$ & $<0.001$ \\
\hline Anxiety & 0.141 & 0.785 & - & 0.503 \\
\hline$P$-value & $\mathbf{0 . 0 0 7}$ & 0.001 & & $<0.001$ \\
\hline Total violence score & - & 0.259 & 0.141 & 0.123 \\
\hline$P$-value & & $<0.001$ & $<0.001$ & 0.018 \\
\hline *Values marked in bold are significant & & & \\
\hline
\end{tabular}

\section{Multivariable analysis}

The first linear regression taking the violence score as the dependent variable showed that higher marital conflicts $(B e t a=0.485)$, abuse by a family member (Beta $=2.988)$, and a higher number of pregnancies (Beta $=1.470$ ) were significantly associated with a higher violence score. Whereas, higher household crowding index (Beta=-1.551) was significantly associated with a lower violence score (Table 2, Model 1).

A second linear regression taking the depression score as the dependent variable showed that higher marital conflicts (Beta = 0.159), financial difficulties (Beta $=2.572)$, a history of child abuse (Beta $=2.546)$, and higher violence scores $($ Beta $=0.121)$ were significantly associated with a higher depression score (Table 2, Model 2).

A third linear regression taking the anxiety score as the dependent variable showed that higher marital conflicts $($ Beta $=0.358)$ and financial difficulties (Beta $=3.859)$ were significantly associated with higher anxiety scores (Table 2, Model 3).

A fourth linear regression taking the stress score as the dependent variable showed that higher marital conflicts $($ Beta $=0.070)$ and financial difficulties (Beta $=1.483$ ) were significantly associated with higher perceived stress scores (Table 2, Model 4). 
Table 2

Multivariable analysis

Model 1: Linear regression model taking the total violence score as the dependent variable

\begin{tabular}{|llllll|}
\hline Variable & Unstandardized Beta & Standardized Beta & $P$ & \multicolumn{2}{l|}{$95 \%$ Confidence Interval } \\
\hline Pregnancy status & -0.848 & -0.038 & 0.405 & -2.851 & 1.154 \\
\hline Marital conflict & 0.485 & 0.524 & $<0.001$ & 0.398 & 0.572 \\
\hline Financial difficulties & 0.069 & 0.006 & 0.898 & -0.998 & 1.137 \\
\hline Number of pregnancies & 1.470 & 0.335 & $<0.001$ & 0.919 & 2.021 \\
\hline Abuse by a family member & $\mathbf{2 . 9 8 8}$ & $\mathbf{0 . 1 3 5}$ & 0.008 & 0.803 & 5.172 \\
\hline Woman monthly income & & & & & 2.924 \\
\hline Low vs no income & 1.120 & 0.081 & 0.223 & -0.685 & 2.540 \\
\hline Intermediate vs no income & 0.782 & 0.061 & 0.382 & -0.977 & 3.000 \\
\hline High vs no income & 1.309 & 0.084 & 0.129 & -0.382 & -0.343
\end{tabular}

Variables entered in the models: Pregnancy status, marital conflict, financial difficulties, duration of marriage, woman age, woman work status, number of pregnancies, number of children, partner smoking status, abuse by a family member, history of child abuse, monthly income and household crowding index.

Model 2: Linear regression model taking the depression scale as the dependent variable and the total violence score as the independent variable

\begin{tabular}{llllll} 
Variable & Unstandardized Beta & Standardized Beta & $P$ & \multicolumn{2}{c}{$95 \%$ Confidence Interval } \\
\hline Pregnancy status & -0.696 & -0.035 & 0.511 & -2.778 & 1.387 \\
\hline Marital conflict & 0.159 & 0.194 & 0.004 & 0.051 & 0.267 \\
\hline Financial difficulties & 2.572 & 0.241 & $<0.001$ & 1.466 & 3.677 \\
\hline History of child abuse & 2.546 & 0.174 & 0.001 & 1.033 & 4.058 \\
\hline Total violence score & 0.121 & 0.137 & 0.045 & 0.003 & 0.240
\end{tabular}

Variables entered in the models: Pregnancy status, marital conflict, financial difficulties, duration of marriage, woman age, woman work status, number of pregnancies, number of children, partner smoking status, abuse by a family member, history of child abuse, monthly income, household crowding index and total violence score.

Model 3: Linear regression model taking the anxiety scale as the dependent variable and the total violence score as the independent variable

\begin{tabular}{lllllc} 
Variable & Unstandardized Beta & Standardized Beta & $P$ & \multicolumn{2}{c}{$95 \%$ Confidence Interval } \\
Pregnancy status & -2.183 & -0.080 & 0.137 & -5.065 & 0.698 \\
\hline Marital conflict & 0.358 & 0.314 & $<0.001$ & 0.209 & 0.508 \\
\hline Financial difficulties & 3.859 & 0.260 & $<0.001$ & $\mathbf{2 . 3 2 9}$ & $\mathbf{5 . 3 8 8}$ \\
\hline Total violence score & 0.018 & 0.014 & 0.833 & -0.146 & 0.181
\end{tabular}

Variables entered in the models: Pregnancy status, marital conflict, financial difficulties, duration of marriage, woman age, woman work status, number of pregnancies, number of children, partner smoking status, abuse by a family member, history of child abuse, monthly income, household crowding index and total violence score

Model 4: Linear regression model taking the stress scale as the dependent variable and the total violence score as the independent variable

Variable

Unstandardized Beta

Standardized Beta

P

95\% Confidence Interval 


\begin{tabular}{|c|c|c|c|c|c|}
\hline \multicolumn{6}{|c|}{ Model 1: Linear regression model taking the total violence score as the dependent variable } \\
\hline Pregnancy status & -0.576 & -0.059 & 0.316 & -1.705 & 0.553 \\
\hline Marital conflict & 0.070 & 0.173 & 0.019 & 0.012 & 0.129 \\
\hline Financial difficulties & 1.483 & 0.280 & $<0.001$ & 0.884 & 2.083 \\
\hline Total violence score & -0.029 & -0.066 & 0.373 & -0.093 & 0.035 \\
\hline \multicolumn{6}{|c|}{$\begin{array}{l}\text { Variables entered in the models: Pregnancy status, marital conflict, financial difficulties, duration of marriage, woman age, } \\
\text { woman work status, number of pregnancies, number of children, partner smoking status, abuse by a family member, history of } \\
\text { child abuse, monthly income, household crowding index and total violence score }\end{array}$} \\
\hline
\end{tabular}

\section{Mediation analysis}

Table 3 presents the mediation analysis conducted on perceived stress, anxiety, and depression. The CASR-SF partially mediated the association between pregnancy status $(25.28 \%)$, financial difficulties $(7.71 \%)$, history of child abuse (21.92\%), abuse by a family member (50.50\%), and anxiety. Also, the CASR-SF partially mediated the association between marital conflicts (21.42\%), financial difficulties $(14.17 \%)$, history of child abuse (20.05\%), abuse by a family member (33.57\%), and depression. No mediation effect of the CASR-SF was found between the independent variables and perceived stress (Table 3). 
Table 3

Mediation analysis

\begin{tabular}{|c|c|c|c|c|c|c|c|c|c|c|}
\hline \multicolumn{11}{|c|}{ Step 1: Taking the anxiety scale as the dependent variable and the composite abuse scale as a mediation factor } \\
\hline \multirow[t]{3}{*}{ Predictors } & \multicolumn{3}{|c|}{ Mediating variable } & \multicolumn{6}{|c|}{ Dependent variables } & \multirow{3}{*}{$\begin{array}{l}\text { Mediating } \\
\text { effect of } \\
\text { composite } \\
\text { abuse } \\
\text { scale }\end{array}$} \\
\hline & \multicolumn{3}{|c|}{ Composite abuse scale } & \multicolumn{3}{|c|}{ Anxiety and abuse scales } & \multicolumn{3}{|c|}{ Anxiety scale } & \\
\hline & Beta & $\mathrm{t}$ & $\mathbf{p}$ & Beta & $\mathrm{t}$ & $\mathbf{P}$ & Beta & $\mathrm{t}$ & p & \\
\hline \multicolumn{11}{|l|}{$\begin{array}{l}\text { Model 1: } \\
\text { taking the } \\
\text { household } \\
\text { crowding } \\
\text { index as the } \\
\text { independent } \\
\text { variable }\end{array}$} \\
\hline $\begin{array}{l}\text { Household } \\
\text { crowding } \\
\text { index }\end{array}$ & $\begin{array}{l}-1.37 \\
{[-2.73 ;-0.01]}\end{array}$ & -1.98 & 0.047 & $\begin{array}{l}1.17 \\
{[-0.53 ; 2.87]}\end{array}$ & 1.35 & 0.17 & $\begin{array}{l}0.78 \\
{[-0.95 ; 2.52]}\end{array}$ & 0.88 & 0.37 & - \\
\hline $\begin{array}{l}\text { Composite } \\
\text { abuse scale }\end{array}$ & & & & $\begin{array}{l}0.28 \\
{[0.15 ; 0.41]}\end{array}$ & 4.31 & $\begin{array}{l}< \\
0.001\end{array}$ & & & & \\
\hline \multicolumn{11}{|l|}{$\begin{array}{l}\text { Model 2: } \\
\text { taking the } \\
\text { marital } \\
\text { conflict } \\
\text { scale as the } \\
\text { independent } \\
\text { variable }\end{array}$} \\
\hline $\begin{array}{l}\text { Marital } \\
\text { conflict } \\
\text { scale }\end{array}$ & $\begin{array}{l}0.53 \\
{[0.45 ; 0.61]}\end{array}$ & 12.50 & $\begin{array}{l}< \\
0.001\end{array}$ & $\begin{array}{l}0.47 \\
{[0.33 ; 0.61]}\end{array}$ & 6.66 & $\begin{array}{l}< \\
0.001\end{array}$ & $\begin{array}{l}0.47 \\
{[0.36 ; 0.59]}\end{array}$ & 8.02 & $\begin{array}{l}< \\
0.001\end{array}$ & - \\
\hline $\begin{array}{l}\text { Composite } \\
\text { abuse scale }\end{array}$ & & & & $\begin{array}{l}0.004 \\
{[-0.14 ; 0.14]}\end{array}$ & 0.05 & 0.95 & & & & \\
\hline \multicolumn{11}{|l|}{$\begin{array}{l}\text { Model 3: } \\
\text { taking the } \\
\text { pregnancy } \\
\text { status as } \\
\text { the } \\
\text { independent } \\
\text { variable }\end{array}$} \\
\hline $\begin{array}{l}\text { Pregnancy } \\
\text { status }\end{array}$ & $\begin{array}{l}-2.88 \\
{[-5.33 ;-0.43]}\end{array}$ & -2.32 & 0.02 & $\begin{array}{l}-3.56 \\
{[-6.69 ; 0.43]}\end{array}$ & -2.24 & 0.02 & $\begin{array}{l}-4.46 \\
{[-7.65 ;-1.27]}\end{array}$ & -2.75 & 0.006 & $25.28 \%$ \\
\hline $\begin{array}{l}\text { Composite } \\
\text { abuse scale }\end{array}$ & & & & $\begin{array}{l}0.31 \\
{[0.16 ; 0.45]}\end{array}$ & 4.24 & $\begin{array}{l}< \\
0.001\end{array}$ & & & & \\
\hline \multicolumn{11}{|l|}{$\begin{array}{l}\text { Model 4: } \\
\text { taking the } \\
\text { financial } \\
\text { difficulties } \\
\text { as the } \\
\text { independent } \\
\text { variable }\end{array}$} \\
\hline $\begin{array}{l}\text { Financial } \\
\text { difficulties }\end{array}$ & $\begin{array}{l}1.46 \\
{[0.27 ; 2.65]}\end{array}$ & 2.41 & 0.01 & $\begin{array}{l}4.28 \\
{[2.84 ; 5.72]}\end{array}$ & 5.85 & $\begin{array}{l}< \\
0.001\end{array}$ & $\begin{array}{l}4.62 \\
{[3.16 ; 6.07]}\end{array}$ & 6.25 & $\begin{array}{l}< \\
0.001\end{array}$ & $7.71 \%$ \\
\hline $\begin{array}{l}\text { Composite } \\
\text { abuse scale }\end{array}$ & & & & $\begin{array}{l}0.22 \\
{[0.10 ; 0.35]}\end{array}$ & 3.61 & 0.0003 & & & & \\
\hline
\end{tabular}




\begin{tabular}{|c|c|c|c|c|c|c|c|c|c|c|}
\hline \multicolumn{11}{|l|}{$\begin{array}{l}\text { Model 5: } \\
\text { taking the } \\
\text { history of } \\
\text { child abuse } \\
\text { as the } \\
\text { independent } \\
\text { variable }\end{array}$} \\
\hline $\begin{array}{l}\text { History of } \\
\text { child abuse }\end{array}$ & $\begin{array}{l}3.20 \\
{[1.58 ; 4.81]}\end{array}$ & 3.89 & 0.001 & $\begin{array}{l}3.33 \\
{[1.27 ; 5.39]}\end{array}$ & 3.18 & 0.001 & $\begin{array}{l}4.07 \\
{[2.02 ; 6.12]}\end{array}$ & 3.91 & 0.001 & $21.92 \%$ \\
\hline $\begin{array}{l}\text { Composite } \\
\text { abuse scale }\end{array}$ & & & & $\begin{array}{l}0.23 \\
{[0.10 ; 0.35]}\end{array}$ & 3.52 & 0.005 & & & & \\
\hline \multicolumn{11}{|l|}{$\begin{array}{l}\text { Model 6: } \\
\text { taking the } \\
\text { history of } \\
\text { family } \\
\text { member } \\
\text { abuse as } \\
\text { the } \\
\text { independent } \\
\text { variable }\end{array}$} \\
\hline $\begin{array}{l}\text { history of } \\
\text { family } \\
\text { member } \\
\text { abuse }\end{array}$ & $\begin{array}{l}6.35 \\
{[4.13 ; 8.58]}\end{array}$ & 5.62 & $\begin{array}{l}< \\
0.001\end{array}$ & $\begin{array}{l}2.95 \\
{[-0.03 ; 5.93]}\end{array}$ & 1.94 & 0.05 & $\begin{array}{l}4.45 \\
{[1.54 ; 7.35]}\end{array}$ & 3.01 & 0.002 & $50.50 \%$ \\
\hline $\begin{array}{l}\text { Composite } \\
\text { abuse scale }\end{array}$ & & & & $\begin{array}{l}0.23 \\
{[0.10 ; 0.36]}\end{array}$ & 3.49 & 0.005 & & & & \\
\hline
\end{tabular}

Step 2: Taking the depression scale as the dependent variable and the composite abuse scale as a mediation factor

\begin{tabular}{lllll} 
Predictors & Mediating variable & Dependent variables & & $\begin{array}{l}\text { Mediating } \\
\text { effect of } \\
\text { composite }\end{array}$ \\
\cline { 2 - 4 } & Composite abuse scale & depression and abuse scales & depression scale & $\begin{array}{l}\text { abuse } \\
\text { scale }\end{array}$
\end{tabular}

$\begin{array}{lllllllll}\text { Beta } & t & p & \text { Beta } & t & P & \text { Beta } & t & p\end{array}$

Model 1:

taking the

household

crowding

index as the

independent

variable

$\begin{array}{lllllllllll}\begin{array}{l}\text { Household } \\ \text { crowding }\end{array} & -1.37 & -1.98 & 0.047 & \begin{array}{l}0.68 \\ {[-0.55 ; 1.92]}\end{array} & 1.09 & 0.27 & \begin{array}{l}0.30 \\ {[-0.98 ; 1.59]}\end{array} & 0.46 & 0.64 & -\end{array}$

index

\section{Composite}

abuse scale

Model 2:

taking the

marital

conflict

scale as the

independent

variable

Marital

conflict

scale
0.53

[0.45;0.61]
0.28

$[0.18 ; 0.37]$

$\begin{array}{ll}5.91< & < \\ 0.001\end{array}$




\begin{tabular}{|c|c|c|c|c|c|c|c|c|c|c|}
\hline $\begin{array}{l}\text { Composite } \\
\text { abuse scale }\end{array}$ & & & & $\begin{array}{l}0.12 \\
{[0.01 ; 0.23]}\end{array}$ & 2.21 & 0.02 & & & & \\
\hline \multicolumn{11}{|l|}{$\begin{array}{l}\text { Model 3: } \\
\text { taking the } \\
\text { pregnancy } \\
\text { status as } \\
\text { the } \\
\text { independent } \\
\text { variable }\end{array}$} \\
\hline $\begin{array}{l}\text { Pregnancy } \\
\text { status }\end{array}$ & $\begin{array}{l}-2.88 \\
{[-5.33 ;-0.43]}\end{array}$ & -2.32 & 0.02 & $\begin{array}{l}-1.47 \\
{[-3.72 ; 0.76]}\end{array}$ & -1.29 & 0.19 & $\begin{array}{l}-2.31 \\
{[-4.65 ; 0.01]}\end{array}$ & -1.95 & 0.051 & - \\
\hline $\begin{array}{l}\text { Composite } \\
\text { abuse scale }\end{array}$ & & & & $\begin{array}{l}0.29 \\
{[0.18 ; 0.39]}\end{array}$ & 5.51 & $\begin{array}{l}< \\
0.001\end{array}$ & & & & \\
\hline \multicolumn{11}{|l|}{$\begin{array}{l}\text { Model 4: } \\
\text { taking the } \\
\text { financial } \\
\text { difficulties } \\
\text { as the } \\
\text { independent } \\
\text { variable }\end{array}$} \\
\hline $\begin{array}{l}\text { Financial } \\
\text { difficulties }\end{array}$ & $\begin{array}{l}1.46 \\
{[0.27 ; 2.65]}\end{array}$ & 2.41 & 0.01 & $\begin{array}{l}2.54 \\
{[1.47 ; 3.60]}\end{array}$ & 5.85 & $\begin{array}{l}< \\
0.001\end{array}$ & $\begin{array}{l}2.90 \\
{[1.81 ; 4.00]}\end{array}$ & 5.21 & $\begin{array}{l}< \\
0.001\end{array}$ & $14.17 \%$ \\
\hline $\begin{array}{l}\text { Composite } \\
\text { abuse scale }\end{array}$ & & & & $\begin{array}{l}0.24 \\
{[0.15 ; 0.33]}\end{array}$ & 5.36 & $\begin{array}{l}<.001 \\
0.00\end{array}$ & & & & \\
\hline \multicolumn{11}{|l|}{$\begin{array}{l}\text { Model 5: } \\
\text { taking the } \\
\text { history of } \\
\text { child abuse } \\
\text { as the } \\
\text { independent } \\
\text { variable }\end{array}$} \\
\hline $\begin{array}{l}\text { History of } \\
\text { child abuse }\end{array}$ & $\begin{array}{l}3.20 \\
{[1.58 ; 4.81]}\end{array}$ & 3.89 & 0.001 & $\begin{array}{l}3.64 \\
{[2.17 ; 5.11]}\end{array}$ & 4.86 & $<.001$ & $\begin{array}{l}4.38 \\
{[2.89 ; 5.86]}\end{array}$ & 5.78 & $<.001$ & $20.05 \%$ \\
\hline $\begin{array}{l}\text { Composite } \\
\text { abuse scale }\end{array}$ & & & & $\begin{array}{l}0.23 \\
{[0.13 ; 0.32]}\end{array}$ & 4.91 & $\begin{array}{l}< \\
0.001\end{array}$ & & & & \\
\hline \multicolumn{11}{|l|}{$\begin{array}{l}\text { Model 6: } \\
\text { taking the } \\
\text { history of } \\
\text { family } \\
\text { member } \\
\text { abuse as } \\
\text { the } \\
\text { independent } \\
\text { variable }\end{array}$} \\
\hline $\begin{array}{l}\text { history of } \\
\text { family } \\
\text { member } \\
\text { abuse }\end{array}$ & $\begin{array}{l}6.35 \\
{[4.13 ; 8.58]}\end{array}$ & 5.62 & $<.001$ & $\begin{array}{l}4.20 \\
{[2.06 ; 6.34]}\end{array}$ & 3.86 & 0.001 & $\begin{array}{l}5.62 \\
{[3.51 ; 7.73]}\end{array}$ & 5.24 & $<.001$ & $33.57 \%$ \\
\hline $\begin{array}{l}\text { Composite } \\
\text { abuse scale }\end{array}$ & & & & $\begin{array}{l}0.22 \\
{[0.12 ; 0.31]}\end{array}$ & 4.61 & $\begin{array}{l}< \\
0.001\end{array}$ & & & & \\
\hline
\end{tabular}

Step 3: Taking the perceived stress scale as the dependent variable and the composite abuse scale as a mediation factor

\begin{tabular}{lllll} 
Predictors & Mediating variable & Dependent variables & $\begin{array}{l}\text { Mediating } \\
\text { effect of }\end{array}$ \\
\cline { 2 - 4 } & Composite abuse scale & Perceived stress and abuse scales & $\begin{array}{l}\text { Perceived stress } \\
\text { scale }\end{array}$ & $\begin{array}{l}\text { composite } \\
\text { abuse } \\
\text { scale }\end{array}$ \\
\hline
\end{tabular}




\begin{tabular}{|c|c|c|c|c|c|c|c|c|c|c|}
\hline \multicolumn{11}{|c|}{ Step 1: Taking the anxiety scale as the dependent variable and the composite abuse scale as a mediation factor } \\
\hline \multicolumn{11}{|l|}{$\begin{array}{l}\text { Model 1: } \\
\text { taking the } \\
\text { household } \\
\text { crowding } \\
\text { index as the } \\
\text { independent } \\
\text { variable }\end{array}$} \\
\hline $\begin{array}{l}\text { Household } \\
\text { crowding } \\
\text { index }\end{array}$ & $\begin{array}{l}-1.37 \\
{[-2.73 ;-0.01]}\end{array}$ & -1.98 & 0.047 & $\begin{array}{l}0.24 \\
{[-0.38 ; 0.87]}\end{array}$ & 0.77 & 0.43 & $\begin{array}{l}0.17 \\
{[-0.44 ; 0.80]}\end{array}$ & 0.56 & 0.57 & - \\
\hline $\begin{array}{l}\text { Composite } \\
\text { abuse scale }\end{array}$ & & & & $\begin{array}{l}0.04 \\
{[0.001 ; 0.09]}\end{array}$ & 2.04 & 0.04 & & & & \\
\hline \multicolumn{11}{|l|}{$\begin{array}{l}\text { Model 2: } \\
\text { taking the } \\
\text { marital } \\
\text { conflict } \\
\text { scale as the } \\
\text { independent } \\
\text { variable }\end{array}$} \\
\hline $\begin{array}{l}\text { Marital } \\
\text { conflict } \\
\text { scale }\end{array}$ & $\begin{array}{l}0.53 \\
{[0.45 ; 0.61]}\end{array}$ & 12.50 & $<.001$ & $\begin{array}{l}0.10 \\
{[0.04 ; 0.15]}\end{array}$ & 3.67 & 0.003 & $\begin{array}{l}0.09 \\
{[0.05 ; 0.14]}\end{array}$ & 4.17 & $<.001$ & - \\
\hline $\begin{array}{l}\text { Composite } \\
\text { abuse scale }\end{array}$ & & & & $\begin{array}{l}-0.009 \\
{[-0.06 ; 0.04]}\end{array}$ & -0.33 & 0.73 & & & & \\
\hline \multicolumn{11}{|l|}{$\begin{array}{l}\text { Model 3: } \\
\text { taking the } \\
\text { pregnancy } \\
\text { status as } \\
\text { the } \\
\text { independent } \\
\text { variable }\end{array}$} \\
\hline $\begin{array}{l}\text { Pregnancy } \\
\text { status }\end{array}$ & $\begin{array}{l}-2.88 \\
{[-5.33 ;-0.43]}\end{array}$ & -2.32 & 0.02 & $\begin{array}{l}-0.81 \\
{[-1.99 ; 0.37]}\end{array}$ & -1.34 & 0.17 & $\begin{array}{l}-0.90 \\
{[-2.07 ; 0.27]}\end{array}$ & -1.50 & 0.13 & - \\
\hline $\begin{array}{l}\text { Composite } \\
\text { abuse scale }\end{array}$ & & & & $\begin{array}{l}0.03 \\
{[-0.02 ; 0.08]}\end{array}$ & 1.08 & 0.27 & & & & \\
\hline \multicolumn{11}{|l|}{$\begin{array}{l}\text { Model 4: } \\
\text { taking the } \\
\text { financial } \\
\text { difficulties } \\
\text { as the } \\
\text { independent } \\
\text { variable }\end{array}$} \\
\hline $\begin{array}{l}\text { Financial } \\
\text { difficulties }\end{array}$ & $\begin{array}{l}1.46 \\
{[0.27 ; 2.65]}\end{array}$ & 2.41 & 0.01 & $\begin{array}{l}1.45 \\
{[0.91 ; 1.98]}\end{array}$ & 5.34 & $\begin{array}{l}< \\
0.001\end{array}$ & $\begin{array}{l}1.49 \\
{[0.96 ; 2.02]}\end{array}$ & 5.55 & $\begin{array}{l}< \\
0.001\end{array}$ & - \\
\hline $\begin{array}{l}\text { Composite } \\
\text { abuse scale }\end{array}$ & & & & $\begin{array}{l}0.03 \\
{[-0.01 ; 0.07]}\end{array}$ & 1.36 & 0.17 & & & & \\
\hline \multicolumn{11}{|l|}{$\begin{array}{l}\text { Model 5: } \\
\text { taking the } \\
\text { history of } \\
\text { child abuse } \\
\text { as the } \\
\text { independent } \\
\text { variable }\end{array}$} \\
\hline $\begin{array}{l}\text { History of } \\
\text { child abuse }\end{array}$ & $\begin{array}{l}3.20 \\
{[1.58 ; 4.81]}\end{array}$ & 3.89 & 0.001 & $\begin{array}{l}0.06 \\
{[-0.70 ; 0.82]}\end{array}$ & 0.15 & 0.87 & $\begin{array}{l}0.20 \\
{[-0.54 ; 0.96]}\end{array}$ & 0.54 & 0.58 & - \\
\hline
\end{tabular}




\begin{tabular}{|c|c|c|c|c|c|c|c|c|c|c|}
\hline $\begin{array}{l}\text { Composite } \\
\text { abuse scale }\end{array}$ & 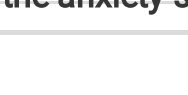 & . & & $\begin{array}{l}0.04 \\
{[-0.001 ; 0.09]}\end{array}$ & 1.90 & 0.058 & & & & \\
\hline \multicolumn{10}{|l|}{$\begin{array}{l}\text { Model 6: } \\
\text { taking the } \\
\text { history of } \\
\text { family } \\
\text { member } \\
\text { abuse as } \\
\text { the } \\
\text { independent } \\
\text { variable }\end{array}$} & \\
\hline $\begin{array}{l}\text { history of } \\
\text { family } \\
\text { member } \\
\text { abuse }\end{array}$ & $\begin{array}{l}6.35 \\
{[4.13 ; 8.58]}\end{array}$ & 5.62 & $<.001$ & $\begin{array}{l}0.14 \\
{[-0.95 ; 1.25]}\end{array}$ & 2.66 & 0.79 & $\begin{array}{l}0.43 \\
{[-0.62 ; 1.49]}\end{array}$ & 0.81 & 0.41 & - \\
\hline $\begin{array}{l}\text { Composite } \\
\text { abuse scale }\end{array}$ & & & & $\begin{array}{l}0.04 \\
{[-0.003 ; 0.09]}\end{array}$ & 1.81 & 0.07 & & & & \\
\hline
\end{tabular}

\section{Discussion}

To our knowledge, this study is the first to assess the factors related to psychological distress among Lebanese women during the COVID-19 confinement. The results showed that marital conflicts and financial difficulties were associated with high stress, anxiety, and depression, while higher violence was related to higher depression among women. Also, violence was a mediating factor between pregnancy status and anxiety and between marital conflict and depression.

In this study, marital conflicts were related to higher depression, anxiety, and stress among women. Consistently, previous findings have also associated high levels of psychological distress with marital dissatisfaction (45-48). A recent study conducted in China during the COVID-19 pandemic showed that emotional well-being in married people was lower than in unmarried people (49). Evidence suggests that stressful or life-threatening events, such as natural disasters, can lead to a decline in the quality of relationships due to stress and conflict or, instead, improve the quality of relationships and intimacy (50-52). Also, during the COVID-19 confinement, several factors can lead to increased tension between romantic partners confined together, such as disturbed everyday activities, the fear of the pandemic, loss of physical activity, loss of outside social networks, lack of access to non-essential health services, and reduced physical interaction in overcrowded households (53). Previous studies have shown that the persistence of tense verbal or non-verbal exchanges between partners can lead to physiological and psychological changes, eventually progressing to disease states $(54,55)$. Once health conditions develop or intensify, they can be experienced as a traumatic life occurrence or persistent stress, resulting in adverse mental health consequences, such as depression and anxiety (56). Our results also showed that marital conflicts were associated with higher violence, consistent with those of previous studies (57-59). The severity of marital conflicts ranges from minor disagreements to disputes that partners cannot overcome, which harms their relationship and could lead to violence (60).

This study showed that violence was associated with higher depression among women, in agreement with previous studies showing that women subject to behavioral, physical, and sexual violence by an intimate partner were more likely to exhibit signs of depression (61-63). The core mechanisms that explain why violence can cause subsequent depression in women are traumatic and psychological stress reactions (64). Sustained psychological stress due to social threats or rejection can affect women's cognition, mood, and behavior, leading to symptoms of depression (65). Also, domestic environments have become a suitable place for intimate partner abuse because of lockdown restrictions (66). Abusive individuals may use the confinement to exert power over the vulnerable ones, especially women (66). Lockdown restrictions can contribute to increased stress and further lead to exacerbation of anxiety, depressive symptoms, and violence (67). Thus, the perpetrator disturbed psychological health could adversely affect that of abused individuals (67). The persistent exposure of vulnerable persons to abusive individuals constrains the victim to cope with the situation, which further aggravates depressive symptoms (68).

Our findings revealed that financial difficulties were associated with higher depression, stress, and anxiety, consistent with those of a recent study showing a correlation between financial hardship and emotional impact due to the pandemic (69). Other studies 
have also reported that financial difficulties due to the lockdown might impair mental health and increase the incidence of psychological stress (70-72). During times of crisis and confinement, women face plenty of problems, including high risks of economic poverty. Also, they are over-represented in health services, continue to do most unpaid household care jobs, and face increased risks of violence, assault, or harassment (73). Additionally, their employment, businesses incomes, and living conditions could be more vulnerable than men to the predicted systemic economic effects of the pandemic, placing them at greater risk of financial instability. All these issues have a considerable impact on the health and psychological well-being of women (73). Psychological difficulties expected by financial pressures include higher psychological distress and intensified issues and relations with family members, friends, and in the workplace (74).

Our results showed that a higher number of pregnancies and abuse by a family member were related to higher violence against women. Similarly, other studies found that a large family is a risk factor for frequent domestic violence, empowered by lower socioeconomic status, deprivation of maternal care, neglect of children, lack of family planning, and parenteral conflict (75-78). Furthermore, a history of child abuse was related to higher depression among women, consistently with other findings (79-82). Hence, the loss of attachment and the absence of a secure life were the main features for women to experience depression (83, 84). Moreover, women victims of childhood abuse are more likely to endure violence later in their life (85-87). Identifying women with a history of childhood abuse and assaults would provide efficient maternal care and increase awareness against domestic violence.

Our results showed that violence mediated the association between pregnancy status, marital conflicts, financial difficulties, and depression and anxiety but did not mediate between these factors and stress. Contrary to our findings, a recent study conducted in Lebanon during the COVID-19 lockdown among 502 adults found that female gender and physical violence at home were associated with higher stress during the pandemic (88). It also showed that a previous higher socioeconomic status was associated with higher stress, while current financial wellness was correlated with lower stress (88). However, a previous study among 215 mothers found that stressful life events were directly linked to psychological distress, particularly depression through domestic violence, while marital and pregnancy statuses were not related to the presence of domestic violence (29). Several other studies found that dissatisfied married women would present a high risk of mental distress (89-91). The sudden disruption of routine due to the COVID-19 outbreak has affected the populations globally, leading to exacerbation of stress in the majority of people. However, the Lebanese people exhibited psychological resilience during the confinement, as they had faced and continue to endure political instabilities, a decline of essential services, in addition to several types of violence (92); they adapt to abuse and work through it more than they oppose it (92). Regarding gender equality in Lebanese society, women continue to face discrimination at numerous levels, such as decision-making structures, socio-cultural values, and security issues. Men are raised in a manner that gives them a sense of authority over women and makes them expect women to recognize their superiority. Thus, unhappy marriages, marital conflicts, and economic insecurity make women more vulnerable to mental problems $(48,60,80)$. Future studies are necessary to determine the psychological distress faced by Lebanese married women, particularly during confinement.

\section{Limitations}

This study has several limitations. Its findings could not be generalized to the entire Lebanese female population due to the small sample size. Also, its cross-sectional design makes it difficult to find causal relations. The study relies on self-report data, which may be biased by memory recall and social desirability effects. A selection bias is also likely because the sample was not randomly chosen but rather obtained using the snowball sampling method. Residual confounding bias is also possible since there could be variables linked to psychological distress that were not measured. The violence scale was not validated in Arabic among women prior to our investigation.

\section{Conclusion}

Our main findings suggest that violence mediated the association between pregnancy status, marital conflicts, financial difficulties, and depression and anxiety but did not mediate between these factors and stress. During the COVID-19 pandemic, reducing the effect of stress in relationships and attempting to solve existing conflicts are essential steps to assist partners and

Page $15 / 21$ 
families to adapt to new and challenging conditions. Further studies are needed to understand better the factors that mediate the relations between domestic violence and mental well-being among Lebanese women.

\section{Abbreviations}

COVID-19: Coronavirus disease of 2019, USD: United States dollar, CASR-SF: The Composite Abuse Scale Revised - Short Form, PSS-4: Perceived stress scale short version, LAS: Lebanese Anxiety Scale, PHQ-9: Patient Health Questionnaire, SPSS: Statistical Package for Social Sciences, Cl: Confidence Interval, SD: Standard deviation.

\section{Declarations}

\section{Ethics approval and consent to participate}

The Psychiatric Hospital of the Cross ethics committee approved the study protocol (HPC-018-2020). Online Informed consent was obtained from each participant involved in this study on the first page of the questionnaire.

\section{Consent for publication}

Not applicable.

\section{Availability of data and materials}

Data can be made available under reasonable request form the corresponding author.

\section{Competing interests}

The authors have nothing to disclose.

\section{Funding}

None.

\section{Authors' contribution}

$\mathrm{CH}$ designed the study; $\mathrm{CH}, \mathrm{DM}, \mathrm{SBM}$, JA drafted the manuscript; $\mathrm{CH}$ and PS carried out the analysis and interpreted the results; $\mathrm{PS}, \mathrm{HS}, \mathrm{DK}, \mathrm{NL}, \mathrm{JA}$ assisted in drafting and reviewing the manuscript; $\mathrm{CH}, \mathrm{DM}, \mathrm{DK}, \mathrm{NL}, \mathrm{JA}$, and SBM were responsible for data collection; HS edited the paper for English language, PS supervised the course of the article. All authors reviewed and approved the final version of the manuscript.

\section{Acknowledgements}

The authors would like to thank all those who participated in this study, and Mrs. Mireille Awad Zeinoun for the research idea and for helping in data collection. Also, special thanks go to the Order of Midwives in Lebanon who helped in the data collection by filling up and spreading the web-based online survey.

\section{References}

1. Organization WH. Investing in mental health. 2003.

2. Organization WH, Department WHOSA, Health WHODoM, Abuse S. Global status report on alcohol 2004: World Health Organization; 2004.

3. McLachlan R, Gilfillan G, Gordon J. Deep and persistent disadvantage in Australia: Productivity Commission Canberra; 2013.

4. Slade J, Teesson W, Burgess P. The mental health of Australians 2: report on the 2007 National Survey of Mental Health and Wellbeing. 2009.

5. Albert PR. Why is depression more prevalent in women? Journal of psychiatry neuroscience: JPN. 2015;40(4):219. 
6. Kazi A, Fatmi Z, Hatcher J, Kadir MM, Niaz U, Wasserman GA. Social environment and depression among pregnant women in urban areas of Pakistan: importance of social relations. Soc Sci Med. 2006;63(6):1466-76.

7. Fu CM, Parahoo K. Causes of depression: perceptions among people recovering from depression. J Adv Nurs. 2009;65(1):101-9.

8. Patel MJ, Shahid M, Riaz M, Kashif W, Ayaz SI, Khan MS, et al. Drug overdose: a wake up call! Experience at a tertiary care centre in Karachi, Pakistan. J Pak Med Assoc. 2008;58(6):298.

9. Lorant V, Croux C, Weich S, Deliège D, Mackenbach J, Ansseau M. Depression and socio-economic risk factors: 7-year longitudinal population study. The British journal of psychiatry. 2007;190(4):293-8.

10. Berenson AB, Breitkopf CR, Wu ZH. Reproductive correlates of depressive symptoms among low-income minority women. Obstetrics Gynecology. 2003;102(6):1310-7.

11. Zuraida Zainal N. Depressive symptoms in middle-aged women in peninsular Malaysia. Asia Pacific Journal of Public Health. 2008;20(4):360-9.

12. Ting NK, Leong TK. Prevalence and factors associated with depression among rural communities in Negeri Sembilan, Peninsular Malaysia. Journal of Scientific Research and Reports. 2014:1689-702.

13. Cheah YK, Azahadi M, Phang SN, Abd Manaf NH. Sociodemographic, Lifestyle, and Health Factors Associated With Depression and Generalized Anxiety Disorder Among Malaysian Adults. Journal of Primary Care Community Health. 2020;11:2150132720921738.

14. Ali A, Hawkins RL, Chambers DA. Recovery from depression among clients transitioning out of poverty. Am J Orthopsychiatry. 2010;80(1):26.

15. Nisar N, Billoo N, Gadit A. Prevalence of depression and the associated risks factors among adult women in a fishing community. Journal-Pakistan Medical Association. 2004;54(10):519-25.

16. Chowdhary N, Patel V. The effect of spousal violence on women's health: Findings from the Stree Arogya Shodh in Goa, India. Journal of postgraduate medicine. 2008;54(4):306.

17. Niaz U. Women's mental health in Pakistan. World psychiatry. 2004;3(1):60.

18. Manaf MRA, Qureshi AM, Lotfizadeh M, Ganasegeran K, Yadav H, Al-Dubai S. Factors associated with anxiety and depression among outpatients in Malaysia: A cross-sectional study. Malaysian Journal of Public Health Medicine. 2016;16(3):181-7.

19. Ferrari G, Agnew-Davies R, Bailey J, Howard L, Howarth E, Peters TJ, et al. Domestic violence and mental health: a crosssectional survey of women seeking help from domestic violence support services. Global health action. 2016;9(1):29890.

20. Bansal P, Chaudhary A, Soni R, Sharma S, Gupta VK, Kaushal P. Depression and anxiety among middle-aged women: A community-based study. Journal of Family Medicine Primary Care. 2015;4(4):576.

21. Watson KT, Roberts NM, Saunders MR. Factors associated with anxiety and depression among African American and White women. ISRN psychiatry. 2012;2012.

22. Cheung T, Yip PS. Depression, anxiety and symptoms of stress among Hong Kong nurses: a cross-sectional study. Int J Environ Res Public Health. 2015;12(9):11072-100.

23. Gong Y, Han T, Chen W, Dib HH, Yang G, Zhuang R, et al. Prevalence of anxiety and depressive symptoms and related risk factors among physicians in China: a cross-sectional study. PloS one. 2014;9(7):e103242.

24. Shishehgar S, Dolatian M, Majd HA, Bakhtiary M. Perceived pregnancy stress and quality of life amongst Iranian women. Global journal of health science. 2014;6(4):270.

25. Ruiz R, Fullerton J. The measurement of stress in pregnancy. Nurs Health Sci. 1999;1(1):19-25.

26. McCormick MC, Brooks-Gunn J, Shorter T, Holmes JH, Wallace CY, Heagarty MC. Factors associated with smoking in lowincome pregnant women: relationship to birth weight, stressful life events, social support, health behaviors and mental distress. J Clin Epidemiol. 1990;43(5):441-8.

27. Lu MC, Chen B. Racial and ethnic disparities in preterm birth: the role of stressful life events. American journal of obstetrics gynecology. 2004;191(3):691-9.

28. Marquis S, Butler E. Practice guidelines for prenatal and postnatal outreach in British Columbia, Canada. Victoria: Ministry for Children and Families; 2001. 
29. Ceballo R, Ramirez C, Castillo M, Caballero GA, Lozoff B. Domestic violence and women's mental health in Chile. Psychology of Women Quarterly. 2004;28(4):298-308.

30. Fridh M, Lindström M, Rosvall M. Experience of physical violence and mental health among young men and women: a population-based study in Sweden. BMC Public Health. 2014;14(1):29.

31. Evans SE, Davies C, DiLillo D. Exposure to domestic violence: A meta-analysis of child and adolescent outcomes. Aggression violent behavior. 2008;13(2):131-40.

32. Adams AE, Tolman RM, Bybee D, Sullivan CM, Kennedy AC. The impact of intimate partner violence on low-income women's economic well-being: The mediating role of job stability. Violence Against Women. 2012;18(12):1345-67.

33. Lelaurain S, Fonte D, Giger J-C, Guignard S, Lo Monaco G. Legitimizing intimate partner violence: The role of romantic love and the mediating effect of patriarchal ideologies. Journal of interpersonal violence. 2018:0886260518818427.

34. THE WORLD BANK. Lebanon - World Bank Open Data. Available at: https://data.worldbank.org/country/LB. [Last Accessed August 22, 2020].

35. El Asmar K, Chaaya M, Phung KT, Atweh S, Ghosn H, Khoury RM, et al. P2-316: PREVALENCE OF DEPRESSION AND ANXIETY AMONG OLDER ADULTS IN LEBANON: PRELIMINARY DATA FROM BEIRUT AND MOUNT LEBANON. Alzheimer's Dement. 2014;10:P595-P.

36. Melki I, Beydoun H, Khogali M, Tamim H, Yunis K. Household crowding index: a correlate of socioeconomic status and interpregnancy spacing in an urban setting. Journal of Epidemiology Community Health. 2004;58(6):476-80.

37. Forthofer MS, Markman HJ, Cox M, Stanley S, Kessler RC. Associations between marital distress and work loss in a national sample. Journal of Marriage and the Family. 1996:597-605.

38. Ford-Gilboe M, Wathen C, Varcoe C, MacMillan H, Scott-Storey K, Mantler T, et al. Development of a Brief Measure of Intimate Partner Violence Experiences: The Composite Abuse Scale (Revised)-Short Form (CASR-SF). Copyright: Creative Commons License; 2016.

39. Cohen S, Kamarck T, Mermelstein R. A global measure of perceived stress. Journal of health and social behavior. 1983:38596.

40. Hallit S, Obeid S, Haddad C, Hallit R, Akel M, Haddad G, et al. Construction of the Lebanese Anxiety Scale (LAS-10): a new scale to assess anxiety in adult patients. International Journal of Psychiatry in Clinical Practice. 2020:1-8.

41. Sawaya $H$, Atoui M, Hamadeh A, Zeinoun $P$, Nahas Z. Adaptation and initial validation of the Patient Health Questionnaire-9 (PHQ-9) and the Generalized Anxiety Disorder-7 Questionnaire (GAD-7) in an Arabic speaking Lebanese psychiatric outpatient sample. Psychiatry research. 2016;239:245-52.

42. Kroenke K, Spitzer RL. The PHQ-9: a new depression diagnostic and severity measure. Psychiatric annals. 2002;32(9):50915.

43. Yzerbyt V, Muller D, Batailler C, Judd CM. New recommendations for testing indirect effects in mediational models: The need to report and test component paths. J Pers Soc Psychol. 2018;115(6):929.

44. MacKinnon DP, Fairchild AJ, Fritz MS. Mediation analysis. Annu Rev Psychol. 2007;58:593-614.

45. Perrin JS. Marital satisfaction and psychological well-being in clinical and non-clinical samples. 2008.

46. Trudel G, Goldfarb M. Marital and sexual functioning and dysfunctioning, depression and anxiety. Sexologies. 2010;19(3):137-42.

47. Choi $\mathrm{H}$, Marks NF. Marital conflict, depressive symptoms, and functional impairment. Journal of Marriage Family. 2008;70(2):377-90.

48. Goldfarb MR, Trudel G, Boyer R, Préville M. Marital relationship and psychological distress: Its correlates and treatments. Sexual relationship therapy. 2007;22(1):109-26.

49. Yang H, Ma J. How an Epidemic Outbreak Impacts Happiness: Factors that Worsen (vs. Protect) Emotional Well-being during the Coronavirus Pandemic. Psychiatry Research. 2020:113045.

50. Cohan CL, Cole SW. Life course transitions and natural disaster: Marriage, birth, and divorce following Hurricane Hugo. J Fam Psychol. 2002;16(1):14. 
51. Fredman SJ, Monson CM, Schumm JA, Adair KC, Taft CT, Resick PA. Associations among disaster exposure, intimate relationship adjustment, and PTSD symptoms: Can disaster exposure enhance a relationship? J Trauma Stress.

2010;23(4):446-51.

52. Marshall EM, Kuijer RG. Weathering the storm? The impact of trauma on romantic relationships. Current opinion in psychology. 2017;13:54-9.

53. Luetke M, Hensel D, Herbenick D, Rosenberg M. Romantic Relationship Conflict Due to the COVID-19 Pandemic and Changes in Intimate and Sexual Behaviors in a Nationally Representative Sample of American Adults. Journal of Sex Marital Therapy. 2020;46(8):747-62.

54. Robles TF, Kiecolt-Glaser JK. The physiology of marriage: Pathways to health. Physiol Behav. 2003;79(3):409-16.

55. McEwen BS. Protective and damaging effects of stress mediators. New England journal of medicine. 1998;338(3):171-9.

56. Williamson GM, Shaffer DR, Parmelee PA, a Parmelee P, Parmelee PM. Physical illness and depression in older adults: A handbook of theory, research, and practice: Springer Science \& Business Media; 2000.

57. Park GR, Park E-J, Jun J, Kim N-S. Association between intimate partner violence and mental health among Korean married women. Public Health. 2017;152:86-94.

58. Burlaka V, Grogan-Kaylor A, Savchuk O, Graham-Bermann SA. The relationship between family, parent, and child characteristics and intimate-partner violence (IPV) among Ukrainian mothers. Psychology of violence. 2017;7(3):469.

59. Garrison MB, Curtis SV. Marital Conflict, Intimate Partner Violence, and Family Preservation. Empirically Based Interventions Targeting Social Problems: Springer; 2019. p. 227 - 45.

60. Fincham FD. Marital conflict: Correlates, structure, and context. Current directions in psychological science. 2003;12(1):23-7.

61. Pico-Alfonso MA, Garcia-Linares MI, Celda-Navarro N, Blasco-Ros C, Echeburúa E, Martinez M. The impact of physical, psychological, and sexual intimate male partner violence on women's mental health: depressive symptoms, posttraumatic stress disorder, state anxiety, and suicide. Journal of women's health. 2006;15(5):599-611.

62. Beydoun HA, Beydoun MA, Kaufman JS, Lo B, Zonderman AB. Intimate partner violence against adult women and its association with major depressive disorder, depressive symptoms and postpartum depression: a systematic review and metaanalysis. Soc Sci Med. 2012;75(6):959-75.

63. Devries KM, Mak JY, Bacchus LJ, Child JC, Falder G, Petzold M, et al. Intimate partner violence and incident depressive symptoms and suicide attempts: a systematic review of longitudinal studies. PLoS Med. 2013;10(5):e1001439.

64. Organization WH. Global and regional estimates of violence against women: prevalence and health effects of intimate partner violence and non-partner sexual violence: World Health Organization; 2013.

65. Slavich GM, Irwin MR. From stress to inflammation and major depressive disorder: a social signal transduction theory of depression. Psychological bulletin. 2014;140(3):774.

66. Joseph SJ, Mishra A, Bhandari SS, Dutta S. Intimate partner violence during the COVID-19 pandemic in India: From psychiatric and forensic vantage points. Asian journal of psychiatry. 2020;54:102279.

67. Telles LE, Valença AM, Barros AJ, da Silva AG. Domestic violence in the COVID-19 pandemic: a forensic psychiatric perspective. Brazilian Journal of Psychiatry. 2020(AHEAD).

68. Mechanic MB, Weaver TL, Resick PA. Mental health consequences of intimate partner abuse: A multidimensional assessment of four different forms of abuse. Violence Against Women. 2008;14(6):634-54.

69. Skapinakis P, Bellos S, Oikonomou A, Dimitriadis G, Gkikas P, Perdikari E, et al. Depression and Its Relationship with Coping Strategies and IIIness Perceptions during the COVID-19 Lockdown in Greece: A Cross-Sectional Survey of the Population. Depression Research and Treatment. 2020;2020.

70. Hamadani JD, Hasan MI, Baldi AJ, Hossain SJ, Shiraji S, Bhuiyan MSA, et al. Immediate impact of stay-at-home orders to control COVID-19 transmission on socioeconomic conditions, food insecurity, mental health, and intimate partner violence in Bangladeshi women and their families: an interrupted time series. The Lancet Global Health. 2020.

71. Dsouza DD, Quadros S, Hyderabadwala ZJ, Mamun MA. Aggregated COVID-19 suicide incidences in India: Fear of COVID-19 infection is the prominent causative factor. Psychiatry Research. 2020:113145. 
72. Bhuiyan Al, Sakib N, Pakpour AH, Griffiths MD, Mamun MA. COVID-19-related suicides in Bangladesh due to lockdown and economic factors: case study evidence from media reports. International Journal of Mental Health and Addiction. 2020.

73. Home 0 . Women at the core of the fight against COVID-19 crisis.

74. Kahn JR, Pearlin LI. Financial strain over the life course and health among older adults. Journal of health social behavior. 2006;47(1):17-31.

75. Guler N. Physical, emotional, sexual, and economic violence by spouse during pregnancy and associated factors. Dokuz Eylul University School of Nursing Electronic Journal. 2010;3:72-7.

76. Malta LA, McDonald SW, Hegadoren KM, Weller CA, Tough SC. Influence of interpersonal violence on maternal anxiety, depression, stress and parenting morale in the early postpartum: a community based pregnancy cohort study. BMC Pregnancy Childbirth. 2012;12(1):153.

77. Das S, Bapat U, More NS, Alcock G, Joshi W, Pantvaidya S, et al. Intimate partner violence against women during and after pregnancy: a cross-sectional study in Mumbai slums. BMC Public Health. 2013;13(1):817.

78. Kouyoumdjian FG, Calzavara LM, Bondy SJ, O'Campo P, Serwadda D, Nalugoda F, et al. Risk factors for intimate partner violence in women in the Rakai Community Cohort Study, Uganda, from 2000 to 2009. BMC Public Health. 2013;13(1):566.

79. Ouellet-Morin I, Fisher HL, York-Smith M, Fincham-Campbell S, Moffitt TE, Arseneault L. Intimate partner violence and newonset depression: A longitudinal study of women's childhood and adult histories of abuse. Depress Anxiety. 2015;32(5):31624.

80. Howard LM, Oram S, Galley H, Trevillion K, Feder G. Domestic violence and perinatal mental disorders: a systematic review and meta-analysis. PLoS Med. 2013;10(5):e1001452.

81. Fonseca-Machado MdO, Alves LC, Freitas PS, Monteiro JCdS, Gomes-Sponholz FA. Mental health of women who suffer intimate partner violence during pregnancy. Investigación y educación en enfermería. 2014;32(2):e19968-e.

82. SAHIN S, íLÇiOĞLU K. ÜNSAL A. Domestic Violence, Depression and Anxiety During Pregnancy. HEAD. 2017;14(3):204.12.

83. Kwako LE, Noll JG, Putnam FW, Trickett PK. Childhood sexual abuse and attachment: An intergenerational perspective. Clin Child Psychol Psychiatry. 2010;15(3):407-22.

84. Whiffen VE, MacIntosh HB. Mediators of the link between childhood sexual abuse and emotional distress: A critical review. Trauma Violence Abuse. 2005;6(1):24-39.

85. Zeglin RJ, DeRaedt MR, Lanthier RP. Does having children moderate the effect of child sexual abuse on depression? Journal of child sexual abuse. 2015;24(6):607-26.

86. Till-Tentschert $U$. The relation between violence experienced in childhood and women's exposure to violence in later life: evidence from Europe. J interpers Violence. 2017;32(12):1874-94.

87. Antoniou E, latrakis G. Domestic violence during pregnancy in Greece. Int J Environ Res Public Health. 2019;16(21):4222.

88. Salameh P, Aline H, Badro DA, Carla A-S, Randa A, Sacre H. Mental health outcomes of the CoViD-19 pandemic and a collapsing economy: perspectives from a developing country. Psychiatry research. 2020:113520.

89. Brock RL, Lawrence E. Marriage as a risk factor for internalizing disorders: Clarifying scope and specificity. J Consult Clin Psychol. 2011;79(5):577.

90. Røsand G-MB, Slinning K, Røysamb E, Tambs K. Relationship dissatisfaction and other risk factors for future relationship dissolution: a population-based study of 18,523 couples. Soc Psychiatry Psychiatr Epidemiol. 2014;49(1):109-19.

91. Lorenz FO, Conger RD, Montague RB, Wickrama K. Economic Conditions, Spouse Support, and Psychological Distress of Rural Husbands and Wives 1. Rural Sociology. 1993;58(2):247-68.

92. International Crisis Group. Lebanon's Self-Defeating Survival Strategies. Available at: https://d2071andvip0wj.cloudfront.net/160-lebanon-s-self-defeating-survival-strategies.pdf. [Last Accessed November 15, 2020]. 2015.

\section{Figures}




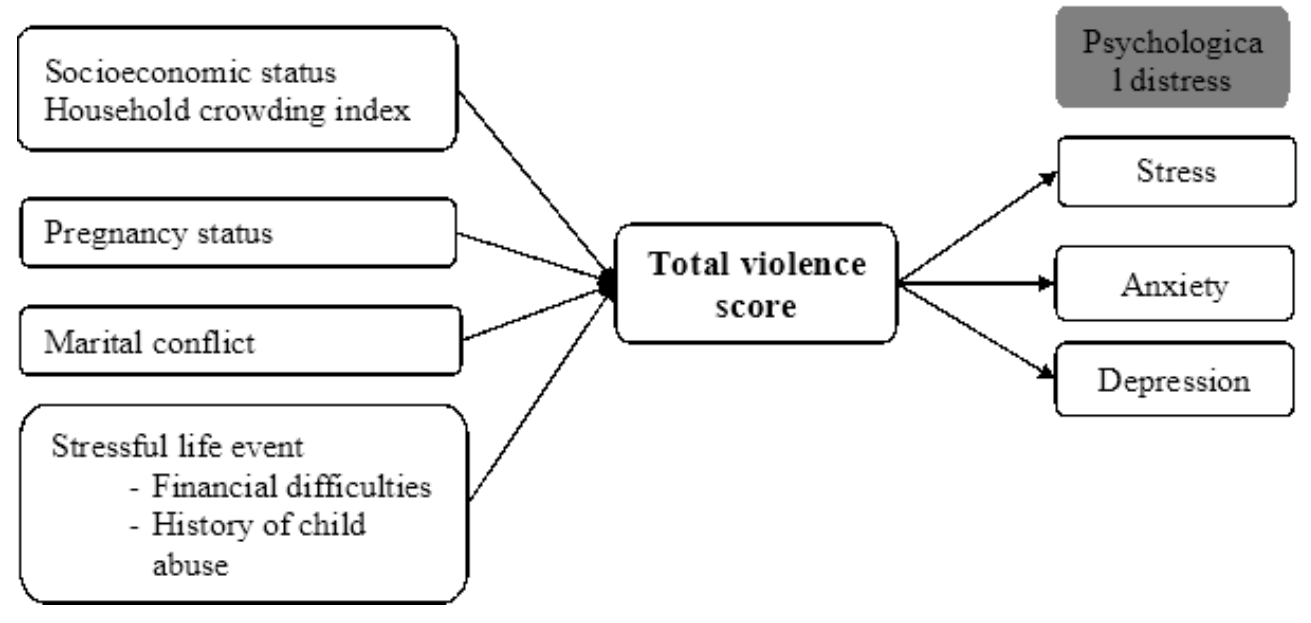

Figure 1

Conceptual model of risk factors associated with violence and the psychological effects among women 